\title{
ON THE DERIVED CATEGORY OF THE CAYLEY PLANE II
}

\author{
DANIELE FAENZI AND LAURENT MANIVEL
}

(Communicated by Lev Borisov)

\begin{abstract}
We find a full strongly exceptional collection for the Cayley plane $\mathbb{O P}^{2}$, the simplest rational homogeneous space of the exceptional group $E_{6}$. This collection, closely related to the one given by the second author in 2011, consists of 27 vector bundles which are homogeneous for the group $E_{6}$, and is a minimal Lefschetz collection with respect to the minimal equivariant embedding of $\mathbb{O} \mathbb{P}^{2}$.
\end{abstract}

\section{INTRODUCTION}

The Cayley plane. Let $X=\mathbb{O P}^{2}$ be the Cayley plane. This is the closed orbit in the projectivization of the minimal representation of the complex simply connected exceptional Lie group $E_{6}$. The Cayley plane can also be identified with the quotient $E_{6} / P_{1}$, where $P_{1}$ is the parabolic subgroup of $E_{6}$ corresponding to the root $\alpha_{1}$ of $E_{6}$. This is sketched in the diagram below, where the simple roots $\alpha_{1}, \ldots, \alpha_{6}$ of $E_{6}$ are depicted. We will denote by $\omega_{1}, \ldots, \omega_{6}$ the corresponding fundamental weights.

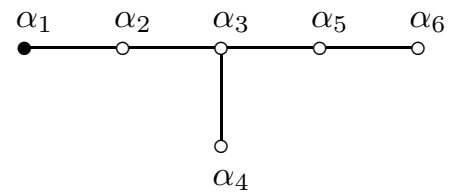

The aim of this note is to provide a full strongly exceptional collection of the derived category of coherent sheaves on the Cayley plane. This completes [Man11, where a strongly exceptional collection closely related to ours was found. But it was not proved that this collection generates the whole derived category.

Our proof of this missing point has two important ingredients. On one hand, we use specific tensor relations between certain homogeneous bundles on the Cayley plane, to show that some of these bundles belong to the category generated by our strongly exceptional collection. This, after a brief account of the geometry of the Cayley plane (Section 1), will be the object of Section 2 This allows us, on the other hand, to use restriction to certain specific subvarieties of the Cayley plane, its maximal quadrics, for which we know a full exceptional collection of the derived category. We will do this in Section 3 , Section 4 contains the proof of our result.

Received by the editors February 24, 2012 and, in revised form, October 30, 2012, April 4, 2013 and July 22, 2013.

2010 Mathematics Subject Classification. Primary 14F05, 14J60, 14 M17.

Key words and phrases. Cayley plane, derived category, full strongly exceptional collection.

The first author was partially supported by ANR projects INTERLOW ANR-09-JCJC-0097-0 and GEOLMI ANR-11-BS03-0011. 
The main result. To state our main result, we need to define two vector bundles $\mathcal{S}$ and $\mathcal{S}_{2}$ on the Cayley plane. The first one is defined as $\mathcal{S}=\mathcal{N}(-1)$, where $\mathcal{N}$ is the normal bundle of the embedding of $X$ in $\mathbb{P}\left(V_{\omega_{1}}\right)$. The second one is defined by the direct sum decomposition (see [Man11]):

$$
S^{2} \mathcal{S}^{*}=\mathcal{S}_{2}^{*} \oplus \mathcal{O}_{X}(-1)
$$

Consider the collections:

$$
\begin{aligned}
& \mathcal{A}=\left(\mathcal{S}_{2}^{*}, \mathcal{S}^{*}, \mathcal{O}_{X}\right), \\
& \mathcal{C}=\left(\mathcal{S}^{*}, \mathcal{O}_{X}\right) .
\end{aligned}
$$

Theorem. The Cayley plane admits the full strongly exceptional collection:

$$
\mathrm{D}^{\mathrm{b}}\left(\mathbb{O P}^{2}\right)=\langle\mathcal{A}, \mathcal{A}(1), \mathcal{A}(2), \mathcal{C}(3), \mathcal{C}(4), \ldots, \mathcal{C}(11)\rangle
$$

This is a Lefschetz collection in the sense of [Kuz07], which is minimal in the sense of [Kuz08. It was used in IM11] to study the derived categories of 7-dimensional cubics.

\section{The geometry of the Cayley plane}

The Cayley plane $X=\mathbb{O P}^{2}$ is a smooth projective variety of dimension 16 and Picard number 1 , homogeneous under the complex exceptional Lie group $E_{6}$. The ample generator $\mathcal{O}_{X}(1)$ of $\operatorname{Pic}(X) \simeq \mathbb{Z}$ embeds $X$ equivariantly as the smallest orbit for $E_{6}$ in the 26-dimensional projective space, acted on linearly by the projectivized smallest non-trivial irreducible $E_{6}$-representation, and we have $\omega_{X} \simeq \mathcal{O}_{X}(-12)$. The Cayley plane is one of the two exceptional complex Hermitian symmetric varieties, and appears naturally as the "last Severi variety", classified by Zak; see Zak93.

1.1. Octonionic plane geometry. The Cayley plane $X=\mathbb{O P}^{2}$ is the complexification of the projective plane over the Cayley octonions. In particular, it contains 8-dimensional quadrics called $\mathbb{O}$-lines, since they can be thought of as copies of the projective line $\mathbb{O P}^{1}$ over the octonions. These $\mathbb{O}$-lines define a (slightly degenerate) plane projective geometry over the octonions. Let us recall briefly the ingredients from this geometry that will be useful in this note, whereby explaining the terminology. For more details, see [M11.

The Cayley plane $X=\mathbb{O P}^{2} \subseteq \mathbb{P}\left(V_{\omega_{1}}\right)$ has to be considered together with its twin $\mathbb{O P}^{2} \subseteq \mathbb{P}\left(V_{\omega_{6}}\right)=\mathbb{P}\left(V_{\omega_{1}}^{*}\right)$. Both varieties are isomorphic as embedded projective varieties, but not equivariantly. If $x$ is a point of $X$, the orthogonal to its tangent space in $\mathbb{P}\left(V_{\omega_{1}}^{*}\right)$ cuts the dual Cayley plane $\mathbb{O P}^{2}$ along a smooth 8-dimensional quadric $\check{Q}_{x}$. The linear span of $\check{Q}_{x}$ is the 10 -dimensional vector space $\mathcal{S}_{x}^{*}$. Symmetrically, each point $\ell$ in $\mathbb{O P}^{2}$ defines a smooth 8-dimensional quadric $Q_{\ell}$ in the Cayley plane. Correspondingly we have a vector bundle $\breve{\mathcal{S}}$ of rank 10 on $\mathbb{O}^{2} \breve{P}^{2}$. The incidence variety $\left\{(x, \ell) \in X \times \mathbb{O P}^{2} \mid x \in Q_{\ell}\right\}$ is the flag variety $E_{6} / P_{1,6}$, and we have:

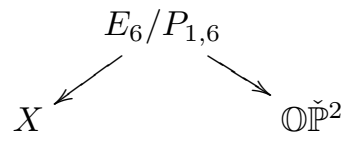

where both maps are fibrations in smooth 8-dimensional quadrics. 
We call these quadrics the $\mathbb{O}$-lines. They have (up to a grain of salt: the word general in the next statements) the characteristic properties of a plane projective geometry: two general $\mathbb{O}$-lines meet in a single point, and through two general points pass a unique $\mathbb{O}$-line. They can also be considered as entry-loci in the following sense. The secant variety of $X$ is a cubic hypersurface in $\mathbb{P}\left(V_{\omega_{1}}\right)$ which we call the Cartan cubic $\mathscr{C}$; see IM11. This cubic is also the dual variety of $\mathbb{O}^{2}{ }^{2}$, so we have a birational map (in fact a resolution of singularities) $\nu: \mathbb{P}\left(\check{\mathcal{S}}^{*}\right) \rightarrow \mathscr{C} \subseteq \mathbb{P}\left(V_{\omega_{1}}\right)$, which is given by the relatively ample line bundle on $\mathbb{P}\left(\check{\mathcal{S}}^{*}\right)$. The flag variety $E_{6} / P_{1,6}$ is the exceptional divisor of $\nu$ in $\mathbb{P}\left(\check{\mathcal{S}}^{*}\right)$.

The secant variety $\mathscr{C}$ is degenerate (and therefore equal to the tangent variety of $X$ ): this means that a point $y$ of the $\mathscr{C} \backslash X$ belongs to infinitely many secant lines to the $\mathscr{C}$. The intersection of these secants with $X$, traditionally called the entry-locus of $y$, is a smooth 8-dimensional quadric, and in fact the $\mathbb{O}$-line $Q_{\ell}$ for some $\ell \in \mathbb{O}^{2}$. Summing up, we have a commutative diagram of $E_{6}$-equivariant morphisms:

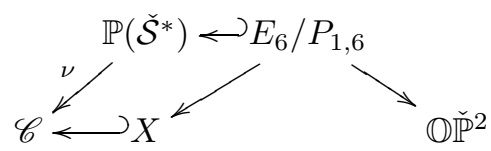

1.2. Homogeneous bundles on the Cayley plane. The category of $E_{6^{-}}$ homogeneous bundles on the Cayley plane $X=\mathbb{O P}^{2}=E_{6} / P_{1}$ is equivalent to the category of $P_{1}$-modules. Let $P_{1}=L P^{u}$ be a Levi decomposition, where $P^{u}$ is the unipotent radical and $L$ is reductive. The center of the Levi factor $L$ is 1-dimensional. The quotient of $L$ by its center is the semisimple part of $P_{1}$, and is isomorphic to $\operatorname{Spin}_{10}$.

The unipotent radical $P^{u}$ has to act trivially on an irreducible $P_{1}$-module, which is therefore completely determined by its $L$-module structure. An irreducible $L$ module is determined by its highest weight, an $L$-dominant weight of $E_{6}$. The set of $L$-dominant of weights is given by linear combinations $\omega=a_{1} \omega_{1}+\cdots+a_{6} \omega_{6}$, with $a_{2}, \ldots, a_{6} \in \mathbb{Z}_{\geq 0}$ and $a_{1} \in \mathbb{Z}$. We denote by $\mathcal{E}_{\omega}$ the $E_{6}$-homogeneous bundle on $X$ corresponding to the irreducible $L$-module determined by the weight $\omega$.

We have $E_{\omega_{1}} \simeq \mathcal{O}_{X}(1)$, the ample generator of $\operatorname{Pic}(X)$. Set $H=c_{1}\left(\mathcal{O}_{X}(1)\right)$. Further recall that we defined $\mathcal{S}=\mathcal{N}(-1)$, so $\mathcal{S}$ is clearly $E_{6}$-homogeneous. Moreover, we can derive the Chern classes of $\mathcal{S}$ and hence of $\mathcal{S}_{2}$ from [IM05]. Some of the invariants of these bundles are:

$$
\begin{array}{llll}
\operatorname{rk}(\mathcal{S})=10, & c_{1}(\mathcal{S})=5 H, & c_{2}(\mathcal{S})=12 H^{2}, & c_{10}(\mathcal{S})=0 . \\
\operatorname{rk}\left(\mathcal{S}_{2}\right)=54, & c_{1}\left(\mathcal{S}_{2}(-1)\right)=0, & c_{2}\left(\mathcal{S}_{2}(-1)\right)=9 H^{2} . &
\end{array}
$$

Now it is clear that $\mathcal{S} \simeq \mathcal{E}_{\omega_{6}}$, since $\mathcal{E}_{\omega_{6}}$ is the only $E_{6}$-homogeneous bundle of rank 10 with the required Chern classes. We will see another way to identify $\mathcal{E}_{\omega_{6}}$ and $\mathcal{N}(-1)$ at the beginning of the next section. Further, we have $\mathcal{S}_{2}=\mathcal{E}_{2 \omega_{6}}$ (see Man11).

By the Borel-Weil theorem $H^{0}(X, \mathcal{S}) \simeq V_{\omega_{6}}^{*} \simeq V_{\omega_{1}}$ and $\mathcal{S}$ is generated by its global sections. In particular for any point $x$ in the Cayley plane the fibre $\mathcal{S}_{x}^{*}$ of the dual bundle can be considered as a subspace of $V_{\omega_{6}}=V_{\omega_{1}}^{*}$, and this subspace is nothing but the linear span of the $\mathbb{O}$-line $\check{Q}_{x}$.

Recall from Man11, the isomorphisms $\mathcal{S}^{*} \simeq \mathcal{S}(-1), \mathcal{S}_{2}^{*} \simeq \mathcal{S}_{2}(-2)$. 
We already mentioned that $\mathcal{S}$ is globally generated, but the fact that $c_{10}(\mathcal{S})=0$ indicates that a general global section $s$ of $\mathcal{S}$ vanishes nowhere. We can thus define a rank- 9 vector bundle $\mathcal{S}_{s}$ on $X$ by the sequence:

$$
0 \rightarrow \mathcal{O}_{X} \stackrel{s}{\rightarrow} \mathcal{S} \rightarrow \mathcal{S}_{s} \rightarrow 0 .
$$

Moreover $c_{1}\left(\mathcal{S}_{s}\right)=c_{1}(\mathcal{S})=5 H$, and of course $\mathcal{S}_{s}$ is again globally generated. The next lemma will play a crucial role in our argument.

Lemma 1. Consider a vector bundle $\mathcal{S}_{s}$ as above and a global section $\sigma$ of $\mathcal{S}_{s}$.

i) If the global section $\sigma$ is general enough, then it vanishes on the disjoint union $\mathscr{Q}_{\sigma}$ of three smooth 7-dimensional quadrics.

ii) Any smooth 7-dimensional quadric contained in $X$ is a component of some $\mathscr{Q}_{\sigma}$.

Proof. A section $s$ of $\mathcal{S}$ is defined by a vector $v \in V_{\omega_{1}}$, and $s$ vanishes at $x \in X$ if and only if $v$ belongs to the affine tangent space $\hat{\mathcal{T}}_{X, x}$. This can happen only if $v$ belongs to the union of the tangent spaces to the Cayley plane, which is nothing but the cone over the Cartan cubic. In particular this cannot happen if $v$ is general: this explains why $c_{10}(\mathcal{S})=0$.

A section $\sigma$ of $\mathcal{S}_{s}$ is defined by a vector $\bar{u} \in V_{\omega_{1}} / \mathbb{C} v$. It vanishes at $x \in X$ if and only if $u$ belongs to the span of $v$ and the affine tangent space $\hat{\mathcal{T}}_{X, x}$. Generically, the projective line generated by $u$ and $v$ cuts the Cartan cubic into three points $y_{1}, y_{2}, y_{3}$, and the embedded tangent space $T_{X, x}$ must contain one of these three points. Suppose that this point is $y_{1}$. Then $x$ must be on the entry-locus of $y_{1}$, a smooth 8-dimensional quadric $Q_{1}$. Moreover $y_{1}$ must belong to $T_{Q_{1}, x}$, which means that $x$ is on the polar hyperplane $H_{1}$ to $y_{1}$ with respect to $Q_{1}$. Finally, $x$ has to belong to the 7-dimensional quadric $Q_{1} \cap H_{1}$. Switching to $y_{2}$ and $y_{3}$ we obtain in this way three 7-dimensional quadrics that constitute the vanishing locus of $\sigma$. These quadrics have to be disjoint since $\mathcal{S}_{s}$ is globally generated so the vanishing locus of $\sigma$ is smooth. The statement (ii) follows.

To check (iii), let $Q$ be a smooth 7-dimensional quadric. Observe that its linear span $\langle Q\rangle$ cannot be contained in $X$, whose maximal linear spaces are only 5-dimensional by LM03, Corollary 7.19]. Since the Cayley plane is cut out by quadrics, this implies that $X \cap\langle Q\rangle=Q$. Note moreover that $\langle Q\rangle$, being the secant variety of $Q$, is contained in the secant variety of $X$. If we choose a point $z$ in $\langle Q\rangle \backslash Q$, its entry-locus $\tilde{Q}$ is an 8-dimensional quadric. Since the line joining $z$ to any point of $Q$ is contained in $\langle Q\rangle$, it meets $Q$ along a scheme of length two, hence $Q$ must be contained in the entry locus of $z$. Therefore $Q$ must be a hyperplane section of $\tilde{Q}$, say $Q=\tilde{Q} \cap H$. Let $y$ be the point of $\langle\tilde{Q}\rangle$ polar to $H$ with respect to $\tilde{Q}$. Since $Q$ is smooth, $y$ does not belong to $\tilde{Q}$, hence not to the Cayley plane either. A general line through $y$ will allow us to define a section $\sigma$ of some $\mathcal{S}_{s}$ such that $Q$ is a component of $\mathscr{Q}_{\sigma}$.

\section{Generating NeW Bundles from our exceptional Collection}

Given the exceptional collection appearing in our main result, we denote by $\mathscr{D}$ the full subcategory of $\mathrm{D}^{\mathrm{b}}(X)$ generated by it:

$$
\mathscr{D}=\langle\mathcal{A}, \mathcal{A}(1), \mathcal{A}(2), \mathcal{C}(3), \mathcal{C}(4), \ldots, \mathcal{C}(11)\rangle,
$$

which means that $\mathscr{D}$ is the smallest triangulated full subcategory of $\mathrm{D}^{\mathrm{b}}(X)$ containing $(\mathcal{A}, \mathcal{A}(1), \mathcal{A}(2), \mathcal{C}(3), \mathcal{C}(4), \ldots, \mathcal{C}(11))$. One may ask, what are the sheaves 
on $X$ that do lie in $\mathscr{D}$ ? Of course, the goal of this note is to answer: all of them. In this section, we will first prove that some particular sheaves lie in $\mathscr{D}$. We will use these sheaves further on, to prove that the orthogonal of $\mathscr{D}$ in $\mathrm{D}^{\mathrm{b}}(X)$ is zero, which amounts to our main result.

One of our tools for getting new bundles from the generators of $\mathscr{D}$ will be the following classical observation. Given a $P_{1}$-module $E$, we can consider it as an $L$-module, and then define a new $P_{1}$-module structure by extending trivially to $P^{u}$. We get the associated graded module $\operatorname{gr}(E)$, which is a direct sum of irreducible $P_{1}$-modules. Moreover, $E$ can be reconstructed from the summands of $\operatorname{gr}(E)$ by a series of extensions. For instance, starting from the normal bundle sequence and the Euler sequence, we can identify $\mathcal{S}=\mathcal{E}_{\omega_{6}} \simeq \mathcal{N}(-1)$ by observing that $\operatorname{gr}\left(V_{\omega_{1}} \otimes \mathcal{O}_{X}\right) \simeq \mathcal{E}_{\omega_{6}} \oplus \mathcal{O}_{S} \oplus \mathcal{E}_{\omega_{4}}(-1)$, and comparing ranks.

If we consider an irreducible bundle $\mathcal{E}_{\omega}$ defined by an $L$-dominant weight $\omega$, the corresponding irreducible $\operatorname{Spin}_{10}$-representation has highest weight $\omega=a_{2} \omega_{2}+$ $\cdots+a_{6} \omega_{6}$. Relations between representations of $\operatorname{Spin}_{10}$ will therefore imply relations between the corresponding homogeneous bundles. For example, the following fact is general: if we consider a fundamental representation corresponding to an extremal node of some Dynkin diagram, its second skew-symmetric power contains the fundamental representation corresponding to the neighbouring node.
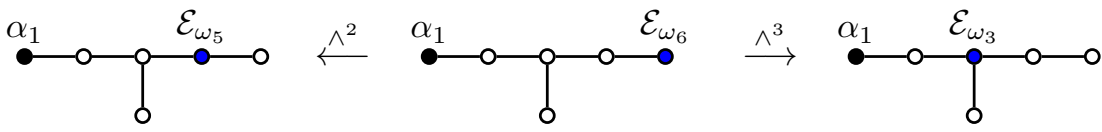

This works well till we attain a triple node or a multiple edge.

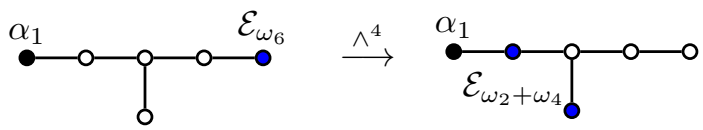

For $\operatorname{Spin}_{10}$ this is particularly neat since these inclusions are in fact always identities. In particular the fundamental representation corresponding to the triple node can be written as a wedge power in three different ways.

To get the correct relations between $L$-modules there remains to adjust the action of the 1-dimensional center, which can be done by computing the first Chern class. Once we cross a triple node, the wedge power contains (and will be equal to, in the cases we will be interested in) the irreducible representation whose highest weight is the sum of the two fundamental weights beyond the triple node (this follows from Theorem 2.1 in [LM04]). This way we get:

Lemma 2. The $E_{6}$-homogeneous bundles defined by the fundamental representations of $L$ can be described as:

$$
\begin{aligned}
& \mathcal{E}_{\omega_{6}} \simeq \mathcal{S}, \quad \mathcal{E}_{\omega_{5}} \simeq \wedge^{2} \mathcal{S}, \quad \mathcal{E}_{\omega_{3}} \simeq \wedge^{3} \mathcal{S}, \\
& \mathcal{E}_{\omega_{4}} \simeq \mathcal{T}_{X}, \quad \mathcal{E}_{\omega_{2}} \simeq \Omega_{X}(2), \quad \mathcal{E}_{\omega_{3}} \simeq \wedge^{2} \mathcal{T}_{X} \simeq \Omega_{X}^{2}(3) .
\end{aligned}
$$

Moreover, the wedge powers that come next are:

$$
\wedge^{4} \mathcal{S} \simeq \mathcal{E}_{\omega_{2}+\omega_{4}}, \quad \wedge^{3} \mathcal{T}_{X} \simeq \mathcal{E}_{\omega_{2}+\omega_{5}}, \quad \Omega_{X}^{3}(4) \simeq \mathcal{E}_{\omega_{4}+\omega_{5}} .
$$

We already mentioned that we also have $\mathcal{E}_{\omega_{1}} \simeq \mathcal{O}_{X}(1)$, the ample generator of the Picard group $\operatorname{Pic}(X) \simeq \mathbb{Z}$. This very ample line bundle defines the natural embedding of $X$ into $\mathbb{P}\left(V_{\omega_{1}}\right)$. One has a natural duality $V_{\omega_{1}}^{*} \simeq V_{\omega_{6}}$, so $H^{0}\left(X, \mathcal{O}_{X}(1)\right) \simeq V_{\omega_{6}}$. Moreover, the bundle $\mathcal{S}(1)$ is the normal bundle of the 
embedding of $X$ into $\mathbb{P}\left(V_{\omega_{1}}\right)$. Let us denote $\Omega_{\mathbb{P}}^{p}=\left.\Omega_{\mathbb{P}\left(V_{\omega_{1}}\right)}^{p}\right|_{X}$ and $\mathcal{T}_{\mathbb{P}}^{p}=\left.\wedge^{p} \mathcal{T}_{\mathbb{P}\left(V_{\omega_{1}}\right)}\right|_{X}$. The Euler sequence reads:

$$
0 \rightarrow \Omega_{\mathbb{P}} \rightarrow V_{\omega_{6}} \otimes \mathcal{O}_{X}(-1) \rightarrow \mathcal{O}_{X} \rightarrow 0,
$$

while the conormal bundle sequence is:

$$
0 \rightarrow \mathcal{S}^{*}(-1) \rightarrow \Omega_{\mathbb{P}} \rightarrow \Omega_{X} \rightarrow 0 .
$$

We get a commutative exact diagram, where we omit zeroes all around the diagram for brevity:

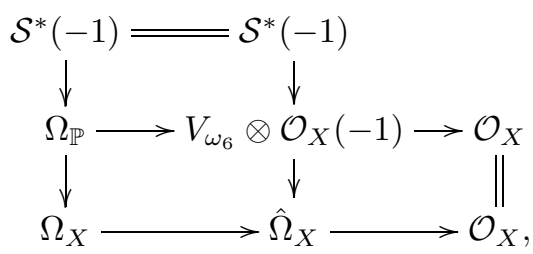

and its dual:

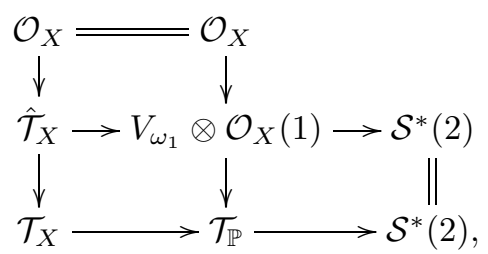

where we denote by $\hat{\mathcal{T}}_{X}$ and $\hat{\Omega}_{X}$ the affine tangent and cotangent bundles of $X$.

Lemma 3. Consider the bundles $F_{1}=\mathcal{T}_{X}(-1) \simeq \mathcal{E}_{\omega_{4}}(-1), F_{2}=\Omega_{X}^{2}(2) \simeq$ $\mathcal{E}_{\omega_{3}}(-1), F_{3}=\mathcal{S}=\mathcal{E}_{\omega_{6}}$, and $F_{4}=\Omega_{X}(2) \simeq \mathcal{E}_{\omega_{2}}$. Then we have:

$$
\begin{aligned}
& \operatorname{Ext}_{X}^{1}\left(F_{3}, F_{1}\right)^{E_{6}} \simeq \operatorname{Ext}_{X}^{1}\left(F_{2}, F_{1}\right)^{E_{6}} \simeq \operatorname{Ext}_{X}^{1}\left(F_{4}, F_{3}\right)^{E_{6}} \simeq \operatorname{Ext}_{X}^{1}\left(F_{4}, F_{2}\right)^{E_{6}} \simeq \mathbb{C}, \\
& \operatorname{Ext}_{X}^{2}\left(F_{4}, F_{1}\right)^{E_{6}} \simeq \mathbb{C},
\end{aligned}
$$

and all other $E_{6}$-invariant components of $\operatorname{Ext}^{i}\left(F_{j}, F_{k}\right)$ are zero, for $i=1,2$ and $j, k=1, \ldots, 4$. Moreover, the Yoneda pairing induces isomorphisms:

$$
\begin{aligned}
& \Upsilon: \operatorname{Ext}_{X}^{1}\left(F_{4}, F_{3}\right)^{E_{6}} \otimes \operatorname{Ext}_{X}^{1}\left(F_{3}, F_{1}\right)^{E_{6}} \rightarrow \operatorname{Ext}_{X}^{2}\left(F_{4}, F_{1}\right)^{E_{6}}, \\
& \Upsilon: \operatorname{Ext}_{X}^{1}\left(F_{4}, F_{2}\right)^{E_{6}} \otimes \operatorname{Ext}_{X}^{1}\left(F_{2}, F_{1}\right)^{E_{6}} \rightarrow \operatorname{Ext}_{X}^{2}\left(F_{4}, F_{1}\right)^{E_{6}} .
\end{aligned}
$$

Proof. Using representations of $\operatorname{Spin}_{10}$ and adjusting the first Chern class, we get:

$$
\begin{aligned}
& \operatorname{gr}\left(F_{1}^{*} \otimes F_{2}\right) \simeq \mathcal{E}_{\omega_{4}} \oplus \mathcal{E}_{\omega_{4}+\omega_{5}}(-1) \oplus \mathcal{E}_{\omega_{2}+\omega_{6}}(-1) \oplus \mathcal{E}_{\omega_{2}+\omega_{3}}(-2), \\
& \operatorname{gr}\left(F_{1}^{*} \otimes F_{3}\right) \simeq \mathcal{E}_{\omega_{4}} \oplus \mathcal{E}_{\omega_{2}+\omega_{6}}(-1), \\
& \operatorname{gr}\left(F_{1}^{*} \otimes F_{4}\right) \simeq \mathcal{E}_{2 \omega_{2}}(-1) \oplus \mathcal{E}_{\omega_{3}} \oplus \mathcal{E}_{\omega_{6}}(1) .
\end{aligned}
$$

For the bundle $F_{2}$ we get:

$$
\begin{aligned}
& \operatorname{gr}\left(F_{2}^{*} \otimes F_{1}\right) \simeq \mathcal{E}_{\omega_{2}}(-2) \oplus \mathcal{E}_{\omega_{4}+\omega_{6}}(-2) \oplus \mathcal{E}_{\omega_{2}+\omega_{5}}(-3) \oplus \mathcal{E}_{\omega_{3}+\omega_{4}}(-3), \\
& \operatorname{gr}\left(F_{2}^{*} \otimes F_{3}\right) \simeq \mathcal{E}_{\omega_{5}}(-1) \oplus \mathcal{E}_{\omega_{2}+\omega_{4}}(-2) \oplus \mathcal{E}_{\omega_{3}+\omega_{6}}(-2), \\
& F_{2}^{*} \otimes F_{4} \simeq F_{1}^{*} \otimes F_{2} .
\end{aligned}
$$


For $F_{3}$, we get:

$$
\begin{aligned}
& \operatorname{gr}\left(F_{3}^{*} \otimes F_{1}\right) \simeq \mathcal{E}_{\omega_{2}}(-2) \oplus \mathcal{E}_{\omega_{4}+\omega_{6}}(-2), \\
& F_{3}^{*} \otimes F_{2} \simeq F_{2}^{*} \otimes F_{3}, \\
& F_{3}^{*} \otimes F_{4} \simeq F_{1}^{*} \otimes F_{3} .
\end{aligned}
$$

Finally for $F_{4}$ :

$$
\begin{aligned}
& \operatorname{gr}\left(F_{4}^{*} \otimes F_{1}\right) \simeq \mathcal{E}_{\omega_{6}}(-2) \oplus \mathcal{E}_{\omega_{3}}(-3) \oplus \mathcal{E}_{2 \omega_{4}}(-3), \\
& F_{4}^{*} \otimes F_{2} \simeq F_{2}^{*} \otimes F_{1}, \\
& F_{4}^{*} \otimes F_{3} \simeq F_{3}^{*} \otimes F_{1} .
\end{aligned}
$$

Now note that the leftmost column of (5) and the bottom row of (6) give nonzero elements in $\operatorname{Ext}_{X}^{1}\left(F_{4}, F_{3}\right)^{E_{6}}$ and $\operatorname{Ext}_{X}^{1}\left(F_{3}, F_{1}\right)^{E_{6}}$. Taking the exterior square of the central column of (5) we get:

$$
0 \rightarrow \Omega_{X}^{2} \rightarrow \hat{\Omega}_{X}^{2} \rightarrow \Omega_{X} \rightarrow 0
$$

which gives a non-zero element $\eta \in \operatorname{Ext}_{X}^{1}\left(F_{4}, F_{2}\right)^{E_{6}}$. Dualizing and using $\mathcal{T}_{X}^{2} \simeq$ $\Omega_{X}^{2}(3)$ (see the previous lemma) we get a non-zero element $\zeta \in \operatorname{Ext}_{X}^{1}\left(F_{2}, F_{1}\right)^{E_{6}}$. Using Bott's theorem and the above decompositions of $\operatorname{gr}\left(F_{j}^{*} \otimes F_{k}\right)$, we get that $\operatorname{Ext}_{X}^{2}\left(F_{4}, F_{1}\right)$ is at most 1-dimensional, and we check that there are no other $E_{6}$ invariant elements in $\operatorname{Ext}^{i}\left(F_{j}, F_{k}\right)$, for $i=1,2$. So it only remains to prove the statement on the non-degeneracy of the Yoneda maps.

For the first map, we apply $\operatorname{Hom}_{X}\left(-, F_{3}(-2)\right)$ to the leftmost column of (5). We get an exact sequence:

$$
\operatorname{Ext}_{X}^{1}\left(\Omega_{\mathbb{P}}(2), F_{3}\right) \rightarrow \operatorname{Ext}_{X}^{1}\left(F_{4}, F_{3}\right) \rightarrow \operatorname{Ext}_{X}^{1}\left(F_{1}, F_{3}\right),
$$

and the rightmost map is $\Upsilon(\zeta \otimes-)$. So, it suffices to prove that $\operatorname{Ext}_{X}^{1}\left(\Omega_{\mathbb{P}}(2), F_{3}\right)=0$. But this is clear by applying $\operatorname{Hom}_{X}\left(-, F_{3}(-2)\right)$ to the central row of (5), since $H^{i}\left(X, F_{3}(-2)\right)=H^{i}\left(X, F_{3}(-1)\right)=0$ for all $i$.

For the second map, we apply $\operatorname{Hom}_{X}\left(-, F_{1}(-2)\right)$ to (9). We get the exact sequence:

$$
\operatorname{Ext}_{X}^{1}\left(\hat{\Omega}_{X}^{2}(2), F_{1}\right) \rightarrow \operatorname{Ext}_{X}^{1}\left(F_{2}, F_{1}\right) \rightarrow \operatorname{Ext}_{X}^{2}\left(F_{4}, F_{1}\right),
$$

and the rightmost map is $\Upsilon(\eta \otimes-)$. So it suffices to check that the leftmost space is zero. Taking the exterior square of the central column of (5) and using (11) we get:

$$
0 \rightarrow \mathcal{S}_{2}(-4) \oplus \mathcal{O}_{X}(-3) \rightarrow V_{\omega_{6}} \otimes \mathcal{S}(-3) \rightarrow V_{\omega_{5}} \otimes \mathcal{O}_{X}(-2) \rightarrow \hat{\Omega}_{X}^{2} \rightarrow 0 .
$$

Note that $H^{1}\left(X, F_{1}\right)=0$ and $\operatorname{Hom}\left(\mathcal{S}(-1), F_{1}\right)=0$ by (8) , since $\operatorname{gr}\left(\mathcal{S} \otimes F_{1}\right)=$ $\operatorname{gr}\left(F_{3}^{*} \otimes F_{1}(1)\right)$. Then, applying $\operatorname{Hom}_{X}\left(-, F_{1}(-2)\right)$ to the above sequence we easily get the desired vanishing $\operatorname{Ext}_{X}^{1}\left(\hat{\Omega}_{X}^{2}(2), F_{1}\right)=0$.

We will simply denote $\Upsilon(a \otimes b)=a b$.

Lemma 4. We have an exact sequence:

$$
\begin{aligned}
0 \rightarrow \mathcal{S}_{2}^{*} \rightarrow V_{\omega_{6}} \otimes \mathcal{S}^{*} & \rightarrow\left(V_{\omega_{1}} \oplus V_{\omega_{5}}\right) \otimes \mathcal{O}_{X} \\
& \rightarrow\left(V_{\omega_{6}} \oplus V_{\omega_{2}}\right) \otimes \mathcal{O}_{X}(1) \rightarrow V_{\omega_{1}} \otimes \mathcal{S}^{*}(2) \rightarrow \mathcal{S}_{2}^{*}(3) \rightarrow 0 .
\end{aligned}
$$

In particular, $\mathcal{S}_{2}^{*}(t)$ lies in $\mathscr{D}$ for $3 \leq t \leq 12$. 
Proof. We start with the exact sequence appearing as the middle row of (6). Taking its symmetric square, using (1), and twisting by $\mathcal{O}_{X}(-1)$ we get:

$$
0 \rightarrow \hat{\mathcal{T}}_{X}^{2}(-1) \rightarrow \wedge^{2} V_{\omega_{1}} \otimes \mathcal{O}_{X}(1) \rightarrow V_{\omega_{1}} \otimes \mathcal{S}^{*}(2) \rightarrow \mathcal{S}_{2}^{*}(3) \oplus \mathcal{O}_{X}(2) \rightarrow 0 .
$$

Let $K$ be the image of the middle map in the above sequence, and note that $\wedge^{2} V_{\omega_{1}} \simeq V_{\omega_{2}}$. Using the surjection $\mathcal{S}_{2}^{*}(3) \oplus \mathcal{O}_{X}(2) \rightarrow \mathcal{S}_{2}^{*}(3)$, we get an epimorphism $V_{\omega_{1}} \otimes \mathcal{S}^{*}(2) \rightarrow \mathcal{S}_{2}^{*}(3)$, whose kernel $\tilde{K}$ fits into:

$$
0 \rightarrow K \rightarrow \tilde{K} \rightarrow \mathcal{O}_{X}(2) \rightarrow 0 \text {. }
$$

By the above sequence, using the middle row of (5) and (10), we get an exact sequence:

$$
0 \rightarrow E \rightarrow\left(V_{\omega_{6}} \oplus V_{\omega_{2}}\right) \otimes \mathcal{O}_{X}(1) \rightarrow V_{\omega_{1}} \otimes \mathcal{S}^{*}(2) \rightarrow \mathcal{S}_{2}^{*}(3) \rightarrow 0,
$$

where $E$ fits into:

$$
0 \rightarrow \hat{\mathcal{T}}_{X}^{2}(-1) \rightarrow E \rightarrow \Omega_{\mathbb{P}}(2) \rightarrow 0 .
$$

Now, we wish to prove that $E \simeq E^{*}(1)$. Note that this will finish the proof, for in this case we can glue (11) with its dual, twisted by $\mathcal{O}_{X}(1)$, to get the desired resolution.

In order to check $E \simeq E^{*}(1)$, first notice that $E$ is exceptional, indeed (11) is nothing but the left mutation of $\mathcal{S}_{2}^{*}(3)$ through $\mathcal{S}^{*}(2)$ and $\mathcal{O}_{X}(1)$, and $\left(\mathcal{O}_{X}(1), \mathcal{S}^{*}(2)\right.$, $\left.\mathcal{S}_{2}^{*}(3)\right)$ is an exceptional collection. In particular, $E$ is indecomposable, and homogeneous for $E_{6}$. Further, note that $E$ fits in the commutative $E_{6}$-invariant exact diagram (where again we omit zeroes all around):

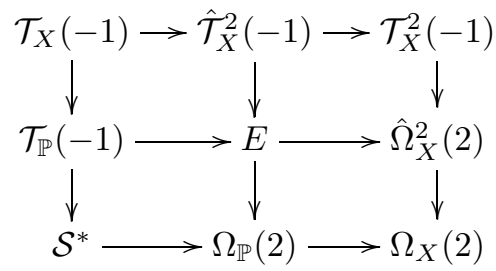

In other words, $\operatorname{gr}(E) \simeq \bigoplus_{i=1}^{4} F_{i}$ in the notation of the previous lemma. Therefore, we are in position to use [OR06, Theorem 5.6], and the bundle $E$ is determined by 4 elements $\left(a_{1}, \ldots, a_{4}\right)$ in $\operatorname{Ext}_{X}^{1}(\operatorname{gr}(E), \operatorname{gr}(E))^{E_{6}}$, where we order the spaces $\operatorname{Ext}_{X}^{1}\left(F_{j}, F_{k}\right)^{E_{6}}$ according to the display of Lemma 3. Moreover, again by Lemma 3 and OR06, Theorem 5.6], we must have $a_{3} a_{1}=a_{4} a_{2}$. This means that $E$ amounts to a representation of the following quiver, with the relation $a_{3} a_{1}=a_{4} a_{2}$ :

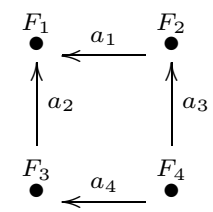

Now, observe that none of the $a_{i}$ is zero. Indeed if for instance $a_{1}=0$, then $a_{3} a_{1}=a_{4} a_{2}$ forces $a_{2}=0$ or $a_{4}=0$, and in both cases $E$ is decomposable. Moreover, note that dualizing (12) and twisting by $\mathcal{O}_{X}(1)$, we get $\operatorname{gr}\left(E^{*}(1)\right) \simeq \operatorname{gr}(E)$, and clearly $E^{*}(1)$ is exceptional too. Then, $E^{*}(1)$ is also given as a representation $\left(b_{1}, \ldots, b_{4}\right)$ of the quiver appearing in (13), where $a_{i}$ is replaced by $b_{i}$ so again $b_{3} b_{1}=b_{4} b_{2}$ and $b_{i} \neq 0$ for all $i$. Therefore, we can find a 4 -tuple $\left(k_{1}, \ldots, k_{4}\right) \in\left(\mathbb{C}^{*}\right)^{4}$, operating on the vertices of our quiver, that takes $\left(b_{1}, \ldots, b_{4}\right)$ to $\left(a_{1}, \ldots, a_{4}\right)$, namely 
$k_{1}=a_{4} a_{2} / b_{2} b_{4}, k_{2}=a_{4} / b_{4}, k_{3}=a_{3} / b_{3}$ and $k_{4}=1$. This says that $E$ and $E^{*}(1)$ correspond to the same representation of our quiver, i.e., $E \simeq E^{*}(1)$.

Lemma 5. The bundle $\wedge^{2} \mathcal{S} \simeq \wedge^{2} \mathcal{S}^{*}(2)$ belongs to the full subcategory of $\mathrm{D}^{\mathrm{b}}(X)$ :

$$
\mathscr{D}_{2}=\left\langle\mathcal{S}^{*}, \mathcal{O}_{X}, \mathcal{O}_{X}(1), \mathcal{O}_{X}(2), \mathcal{S}^{*}(3)\right\rangle \text {. }
$$

In particular,

$$
\wedge^{2} \mathcal{S}^{*}(t) \in \mathscr{D}, \quad \text { for } 2 \leq t \leq 10
$$

Proof. Consider the adjoint representation $V_{\omega_{4}}$. We can compute its restriction to Spin $_{10}$ by the following simple argument. We observe that highest weights for the action of $\operatorname{Spin}_{10}$ are given by the highest roots with a fixed coefficient $k$ on the simple root $\alpha_{1}$. This coefficient $k$ can be equal to 0 or \pm 1 ; for $k=0$ we get the adjoint representation of $\operatorname{Spin}_{10}$ and for $k= \pm 1$ we get the two spin representations. Since $45+16+16=77=78-1$ there is only one trivial factor missing. Taking into account the action of the center of the Levi factor $L$ of $P_{1}$, that is, adjusting the first Chern class, we obtain:

$$
\operatorname{gr}\left(V_{\omega_{4}} \otimes \mathcal{O}_{X}\right) \simeq \mathcal{T}_{X} \oplus \Omega_{X} \oplus \wedge^{2} \mathcal{S}(-1) \oplus \mathcal{O}_{X}
$$

Now, using (5) and (6) we get that $\mathcal{T}_{X}$ belongs to $\left\langle\mathcal{O}_{X}, \mathcal{O}_{X}(1), \mathcal{S}^{*}(2)\right\rangle$ and $\Omega_{X}$ to $\left\langle\mathcal{S}^{*}(-1), \mathcal{O}_{X}(-1), \mathcal{O}_{X}\right\rangle$, so that (14) ensures that $\wedge^{2} \mathcal{S}(-1) \simeq \wedge^{2} \mathcal{S}^{*}(1)$ lies in $\left\langle\mathcal{S}^{*}(-1), \mathcal{O}_{X}(-1), \mathcal{O}_{X}, \mathcal{O}_{X}(1), \mathcal{S}^{*}(2)\right\rangle$. This clearly implies our statement.

Lemma 6. The bundle $\wedge^{3} \mathcal{S} \simeq \wedge^{3} \mathcal{S}^{*}(3)$ lies in:

$$
\mathscr{D}_{3}=\left\langle\mathcal{O}_{X}, \mathcal{S}_{2}^{*}(1), \mathcal{S}^{*}(1), \mathcal{O}_{X}(1), \mathcal{S}^{*}(2), \mathcal{O}_{X}(2), \mathcal{O}_{X}(3)\right\rangle .
$$

In particular,

$$
\wedge^{3} \mathcal{S}^{*}(t) \in \mathscr{D}, \quad \text { for } 3 \leq t \leq 11 .
$$

Proof. We have to check that $\wedge^{3} \mathcal{S} \simeq \Omega_{X}^{2}(3)$ (by Lemma 2) lies in $\mathscr{D}_{3}$. To do this, we take the exterior square of the conormal sequence (4), and we twist by $\mathcal{O}_{X}(3)$, thus obtaining an exact sequence:

$$
0 \rightarrow S^{2} \mathcal{S}^{*}(1) \rightarrow \mathcal{S}^{*} \otimes \Omega_{\mathbb{P}}(2) \rightarrow \Omega_{\mathbb{P}}^{2}(3) \rightarrow \wedge^{3} \mathcal{S}(3) \rightarrow 0 .
$$

We are now led to ask if $S^{2} \mathcal{S}^{*}(1), \mathcal{S}^{*} \otimes \Omega_{\mathbb{P}}(2)$ and $\Omega_{\mathbb{P}}^{2}(3)$ belong to $\mathscr{D}_{3}$.

For the first bundle, recall from (11) the isomorphism $S^{2} \mathcal{S}^{*}(1) \simeq \mathcal{S}_{2}^{*}(1) \oplus \mathcal{O}_{X}$. For the second one, twisting by $\mathcal{S}^{*}$ the Euler sequence (3) we see that $\mathcal{S}^{*} \otimes \Omega_{\mathbb{P}}(2)$ belongs to $\left\langle\mathcal{S}^{*}(1), \mathcal{S}^{*}(2)\right\rangle$. For the third bundle, we take the exterior square of the Euler sequence:

$$
0 \rightarrow \Omega_{\mathbb{P}}^{2} \rightarrow \wedge^{2} V_{\omega_{6}} \otimes \mathcal{O}_{X}(-2) \rightarrow V_{\omega_{6}} \otimes \mathcal{O}_{X}(-1) \rightarrow \mathcal{O}_{X} \rightarrow 0
$$

This readily says that $\Omega_{\mathbb{P}}^{2}(3)$ lies in $\left\langle\mathcal{O}_{X}(1), \mathcal{O}_{X}(2), \mathcal{O}_{X}(3)\right\rangle$. We get $\wedge^{3} \mathcal{S} \in \mathscr{D}_{3}$.

Using the isomorphism $\wedge^{3} \mathcal{S}^{*} \simeq \wedge^{3} \mathcal{S}(-3)$, and Lemma 4, we deduce that $\wedge^{3} \mathcal{S}^{*}(t)$ lies in $\mathscr{D}$, for $3 \leq t \leq 11$.

Lemma 7. The bundle $\wedge^{4} \mathcal{S} \simeq \wedge^{4} \mathcal{S}^{*}(4)$ lies in:

$$
\mathscr{D}_{4}=\left\langle\mathcal{S}^{*}(1), \mathcal{O}_{X}(1), \mathcal{S}^{*}(2), \mathcal{O}_{X}(2), \mathcal{S}_{2}^{*}(3), \mathcal{S}^{*}(3), \mathcal{O}_{X}(3), \mathcal{S}^{*}(4)\right\rangle .
$$

In particular,

$$
\wedge^{4} \mathcal{S}^{*}(t) \in \mathscr{D}, \quad \text { for } 3 \leq t \leq 11
$$


Proof. By Lemma 2, we have $\wedge^{4} \mathcal{S} \simeq \mathcal{E}_{\omega_{2}+\omega_{4}}$. Using the same method as before we observe that the completely irreducible bundle associated to $\mathcal{E}_{\omega_{2}} \otimes \mathcal{E}_{\omega_{4}} \simeq \Omega_{X}(2) \otimes \mathcal{T}_{X}$ is the following:

$$
\operatorname{gr}\left(\mathcal{E}_{\omega_{2}} \otimes \mathcal{E}_{\omega_{4}}\right)=\mathcal{E}_{\omega_{2}+\omega_{4}} \oplus \wedge^{2} \mathcal{S}(1) \oplus \mathcal{O}_{X}(2) .
$$

Therefore, it will be enough to show that the three bundles $\Omega_{X}(2) \otimes \mathcal{T}_{X}, \wedge^{2} \mathcal{S}(1)$ and $\mathcal{O}_{X}(2)$ lie in the subcategory $\mathscr{D}_{4}$. This is obviously the case for $\mathcal{O}_{X}(2)$, and also for $\wedge^{2} \mathcal{S}(1)$ in view of Lemma 5 . To show that $\Omega_{X}(2) \otimes \mathcal{T}_{X}$ lies in $\mathscr{D}_{4}$, we first consider the complexes lying on the diagonal of (5), twisted by $\mathcal{O}_{X}(2)$, together with its dual, lying on the diagonal of ([6). Tensoring these two complexes, we get a third complex of the form:

$$
\begin{aligned}
& \mathcal{S}^{*} \otimes \mathcal{S}^{*}(3)
\end{aligned}
$$

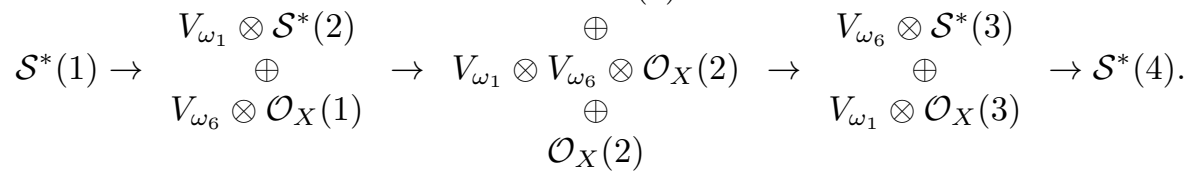

The proof will be finished once we show that all the terms appearing in this complex lie in $\mathscr{D}_{4}$. This is clear for the twists of $\mathcal{O}_{X}$ and of $\mathcal{S}^{*}$. Note that the only term which is not of this form is:

$$
\mathcal{S}^{*} \otimes \mathcal{S}^{*}(3) \simeq \wedge^{2} \mathcal{S}^{*}(3) \oplus \mathcal{S}_{2}^{*}(3) \oplus \mathcal{O}_{X}(2) ;
$$

see (11). But we have already seen that $\wedge^{2} \mathcal{S}^{*}(3) \simeq \wedge^{2} \mathcal{S}(1)$ lies in $\mathscr{D}_{4}$, as well as $\mathcal{S}_{2}^{*}(3)$ and $\mathcal{O}_{X}(2)$, so the lemma is proved.

Set $\mathcal{S}_{3}=\mathcal{E}_{3 \omega_{1}}$ and recall from Man11] that $S^{3} \mathcal{S} \simeq \mathcal{S}_{3} \oplus \mathcal{S}(1)$ and $\mathcal{S}_{3}^{*} \simeq \mathcal{S}_{3}(-3)$.

Lemma 8. The bundle $\mathcal{S}_{3}^{*}(t)$ lies in $\mathscr{D}$ for $1 \leq t \leq 6$.

Proof. Taking the symmetric cube of (4) twisted by $\mathcal{O}_{X}(3)$, we get:

$$
0 \rightarrow S^{3} \mathcal{S}^{*} \rightarrow S^{2} \mathcal{S}^{*} \otimes \Omega_{\mathbb{P}}(1) \rightarrow \mathcal{S}^{*} \otimes \Omega_{\mathbb{P}}^{2}(2) \rightarrow \Omega_{\mathbb{P}}^{3}(3) \rightarrow \Omega_{X}^{3}(3) \rightarrow 0 .
$$

We look at the various terms of this sequence. The first one decomposes as $\mathcal{S}^{*}(-1) \oplus$ $\mathcal{S}_{3}^{*}$, and our aim is to understand which of its twists lie in $\mathscr{D}$, so let us study the other terms. The second term lies in $\left\langle\mathcal{O}_{X}(-1), \mathcal{S}_{2}^{*}, \mathcal{O}_{X}, \mathcal{S}_{2}^{*}(1)\right\rangle$, the third one in $\left\langle\mathcal{S}^{*}, \mathcal{S}^{*}(1), \mathcal{S}^{*}(2)\right\rangle$, and the fourth one in $\left\langle\mathcal{O}_{X}, \mathcal{O}_{X}(1), \mathcal{O}_{X}(2), \mathcal{O}_{X}(3)\right\rangle$. So it remains to study the last term $\Omega_{X}^{3}(3)$.

By Lemma 2 we know that $\Omega_{X}^{3}(3) \simeq \mathcal{E}_{\omega_{4}+\omega_{5}}(-1)$. Moreover we check that:

$$
\begin{aligned}
& \operatorname{gr}\left(\mathcal{E}_{\omega_{4}} \otimes \mathcal{E}_{\omega_{5}}(-1)\right) \simeq \mathcal{E}_{\omega_{4}+\omega_{5}}(-1) \oplus \mathcal{E}_{\omega_{2}+\omega_{6}}(-1) \oplus \mathcal{E}_{\omega_{4}}, \\
& \operatorname{gr}\left(\mathcal{E}_{\omega_{2}} \otimes \mathcal{E}_{\omega_{6}}(-1)\right) \simeq \mathcal{E}_{\omega_{2}+\omega_{6}}(-1) \oplus \mathcal{E}_{\omega_{4}},
\end{aligned}
$$

where by the way the second formula is just (7). We deduce that $\Omega_{X}^{3}(3)$ lies in the full subcategory of $\mathrm{D}^{\mathrm{b}}(X)$ generated by $\mathcal{E}_{\omega_{4}} \otimes \mathcal{E}_{\omega_{5}}(-1), \mathcal{E}_{\omega_{2}} \otimes \mathcal{E}_{\omega_{6}}(-1)$ and $\mathcal{E}_{\omega_{4}}$. Some of these terms can be settled rather easily, for instance

$$
\mathcal{E}_{\omega_{4}} \simeq \mathcal{T}_{X} \in\left\langle\mathcal{O}_{X}, \mathcal{O}_{X}(1), \mathcal{S}^{*}(2)\right\rangle
$$

by (6). Next, $\mathcal{E}_{\omega_{6}}(-1) \simeq \mathcal{S}^{*}$ and

$$
\mathcal{E}_{\omega_{2}} \simeq \Omega_{X}(2) \in\left\langle\mathcal{S}^{*}(1), \mathcal{O}_{X}(1), \mathcal{O}_{X}(2)\right\rangle,
$$

by (5), so $\mathcal{E}_{\omega_{2}} \otimes \mathcal{E}_{\omega_{6}}(-1)$ lies in $\left\langle\wedge^{2} \mathcal{S}^{*}(1), \mathcal{O}_{X}, \mathcal{S}_{2}^{*}(1), \mathcal{S}^{*}(1), \mathcal{S}^{*}(2)\right\rangle$, so Lemma 5 will take care of this term. 
It remains to analyze the summand $\mathcal{E}_{\omega_{4}} \otimes \mathcal{E}_{\omega_{5}}(-1)$. First recall by Lemma 2 that this bundle is isomorphic to $\mathcal{T}_{X} \otimes \wedge^{2} \mathcal{S}^{*}(1)$. Moreover, $\mathcal{T}_{X} \in\left\langle\mathcal{O}_{X}, \mathcal{O}_{X}(1), \mathcal{S}^{*}(2)\right\rangle$, and by Lemma $5 \wedge^{2} \mathcal{S}^{*}(1)$ belongs to $\left\langle\mathcal{S}^{*}(-1), \mathcal{O}_{X}(-1), \mathcal{O}_{X}, \mathcal{O}_{X}(1), \mathcal{S}^{*}(2)\right\rangle$. Recalling that $\mathcal{S}^{*} \otimes \mathcal{S}^{*} \simeq \mathcal{S}_{2}^{*} \oplus \wedge^{2} \mathcal{S}^{*} \oplus \mathcal{O}_{X}(-1)$ we deduce that $\mathcal{T}_{X} \otimes \wedge^{2} \mathcal{S}^{*}(1)$ belongs to:

$$
\left\langle\wedge^{2} \mathcal{S}^{*}(1), \mathcal{S}_{2}^{*}(1),\left\{\mathcal{S}^{*}(r), \mathcal{O}_{X}(s), \text { for }-1 \leq r, s \leq 3\right\}, \mathcal{S}_{2}^{*}(4), \wedge^{2} \mathcal{S}^{*}(4)\right\rangle \text {. }
$$

Putting all these elements together, we get that $\mathcal{S}_{3}^{*}$ lies in:

$$
\left\langle\left\{\mathcal{S}_{2}^{*}(p), \mathcal{S}^{*}(r), \mathcal{O}_{X}(s) \mid 0 \leq p \leq 4,-1 \leq r \leq 3,-1 \leq s \leq 3\right\}, \wedge^{2} \mathcal{S}^{*}(1), \wedge^{2} \mathcal{S}^{*}(4)\right\rangle .
$$

Using Lemma 5 and Lemma 4, we deduce that $\mathcal{S}_{3}^{*}(t)$ lies in $\mathscr{D}$ for $1 \leq t \leq 6$.

One can deduce from these lemmas that many more bundles lie in $\mathscr{D}$. One example of this is the next result, that we will need further on.

Corollary 9. We have:

$$
\begin{array}{ll}
\wedge^{2} \mathcal{S}^{*} \otimes \mathcal{S}^{*}(t) \in \mathscr{D}, & \text { for } 2 \leq t \leq 9, \\
\wedge^{3} \mathcal{S}^{*} \otimes \mathcal{S}^{*}(t) \in \mathscr{D}, & \text { for } 5 \leq t \leq 8, \\
\wedge^{4} \mathcal{S}^{*} \otimes \mathcal{S}^{*}(t) \in \mathscr{D}, & \text { for } 5 \leq t \leq 7 .
\end{array}
$$

Proof. In order to prove (15), first recall that by Lemma 5, we know that $\wedge^{2} \mathcal{S}^{*}(2) \in$ $\left\langle\mathcal{S}^{*}, \mathcal{O}_{X}, \mathcal{O}_{X}(1), \mathcal{O}_{X}(2), \mathcal{S}^{*}(3)\right\rangle$. We deduce that $\wedge^{2} \mathcal{S}^{*} \otimes \mathcal{S}^{*}(4)$ lies in:

$$
\left\langle\wedge^{2} \mathcal{S}^{*}(2), \mathcal{O}_{X}(1), \mathcal{S}_{2}^{*}(2), \mathcal{S}^{*}(2), \mathcal{S}^{*}(3), \mathcal{S}^{*}(4), \mathcal{O}_{X}(4), \mathcal{S}_{2}^{*}(5), \wedge^{2} \mathcal{S}^{*}(5)\right\rangle .
$$

Therefore $\wedge^{2} \mathcal{S}^{*} \otimes \mathcal{S}^{*}(t) \in \mathscr{D}$ for $4 \leq t \leq 9$.

Now we use the relation:

$$
\wedge^{2} \mathcal{S}^{*} \otimes \mathcal{S}^{*} \oplus \mathcal{S}_{3}^{*} \simeq \mathcal{S}_{2}^{*} \otimes \mathcal{S}^{*} \oplus \wedge^{3} \mathcal{S}^{*}
$$

which is easy to establish using $\operatorname{Spin}_{10}$-representations. With Lemmas 8 and 6 this implies that $\mathcal{S}_{2}^{*} \otimes \mathcal{S}^{*}(t) \in \mathscr{D}$ for $4 \leq t \leq 6$. Then we can use the complex from Lemma 4 twisted by $\mathcal{S}^{*}$, and deduce that this is still true for $t=2,3$. Then $\wedge^{2} \mathcal{S}^{*} \otimes \mathcal{S}^{*}(t)$ also belongs to $\mathscr{D}$ for $t=2,3$.

To prove (16), we use Lemma 6, and we tensor by $\mathcal{S}^{*}(-3)$ the generators of the subcategory $\mathscr{D}_{3}$ appearing in this lemma. We find that $\wedge^{3} \mathcal{S}^{*} \otimes \mathcal{S}^{*}$ lies in the subcategory generated by $\mathcal{S}^{*}(r)$, with $-3 \leq r \leq 0$ and by $\mathcal{S}^{*} \otimes \mathcal{S}^{*}(-2), \mathcal{S}^{*} \otimes \mathcal{S}^{*}(-1)$, $\mathcal{S}_{2}^{*} \otimes \mathcal{S}^{*}(-2)$. All these terms have been previously encountered. Using the relation (18), Lemma 8. Lemma 5 and Lemma 4 we get (16).

It remains to prove (17). We use Lemma 7 to see that:

$$
\wedge^{4} \mathcal{S}^{*} \otimes \mathcal{S}^{*} \in\left\langle\wedge^{2} \mathcal{S}^{*}(q), \mathcal{S}_{2}^{*}(p), \mathcal{S}^{*}(r), \mathcal{O}_{X}(s)\right\rangle,
$$

with $-3 \leq q, p \leq 0,-3 \leq r \leq-1$, and $-4 \leq s \leq-1$. Now the relation (17) can be deduced from Lemmas 4 , 5 and 8

\section{Restrictions to QUADRICS OF DIMENSION SEVEN AND EIGHT}

In order to prove our main result, our strategy will consist of restricting to quadrics in the Cayley plane: either $\mathbb{O}$-lines, which are 8-dimensional, or the 7dimensional quadrics that appear in Lemma 1. In this section we prove that being orthogonal to $\mathscr{D}$ imposes drastic conditions on the restrictions to such quadrics. The final conclusion that ${ }^{\perp} \mathscr{D}=0$ will be obtained in the last section.

The following lemma, certainly known to experts, closely resembles OSS80, Theorem 2.3.2]. We provide the proof for the reader's convenience. 
Let us denote by $Q_{n}$ a smooth quadric hypersurface in $\mathbb{P}^{n+1}$. If $n$ is odd, we denote by $\Sigma$ the spinor bundle on $Q_{n}$. If $n$ is even, we denote by $\Sigma_{+}$and $\Sigma_{-}$the two spinor bundles on $Q_{n}$ (see [0tt88]).

Lemma 10. Let $n \geq 3$ and let $E$ be a vector bundle on $Q_{n}$. Then $E$ splits as $\bigoplus_{i} \mathcal{O}_{Q_{n}}\left(a_{i}\right)$ if and only if the restriction of $E$ to some quadric surface $Q_{2} \subseteq Q_{n}$ splits as $\bigoplus_{i} \mathcal{O}_{Q_{2}}\left(a_{i}\right)$.

Proof. One direction is obvious, and it suffices to prove that $E$ splits as $\bigoplus_{i} \mathcal{O}_{Q_{n}}\left(a_{i}\right)$ if the restriction $\left.E\right|_{Q_{n-1}}$ to a codimension-one quadric splits as $\bigoplus_{i} \mathcal{O}_{Q_{n-1}}\left(a_{i}\right)$, for $n \geq 3$. Consider the exact sequence:

$$
\left.0 \rightarrow E(t-1) \rightarrow E(t) \rightarrow E\right|_{Q_{n-1}}(t) \rightarrow 0 .
$$

Fix $t \in \mathbb{Z}$. Since the restricted bundle $\left.E\right|_{Q_{n-1}}$ splits as $\bigoplus_{i} \mathcal{O}_{Q_{n-1}}\left(a_{i}\right)$, and $n \geq 3$, we have $H^{1}\left(Q_{n-1},\left.E\right|_{Q_{n-1}}(t)\right)=0$ so $H^{1}\left(Q_{n}, E(t-1)\right)$ maps onto $H^{1}\left(Q_{n}, E(t)\right)$. Moreover, since $E$ is locally free we must have $H^{1}\left(Q_{n}, E(t)\right)=0$ for $t \ll 0$. This gives $H^{1}\left(Q_{n}, E(t)\right)=0$ for all $t \in \mathbb{Z}$.

Consider now the bundle $F=\bigoplus_{i} \mathcal{O}_{Q_{n}}\left(a_{i}\right)$. Clearly, we have an epimorphism $\left.F \rightarrow E\right|_{Q_{n-1}}$. Applying $\operatorname{Hom}_{Q_{n}}(F,-)$ to (19) for $t=0$, we see that this epimorphism lifts to a morphism $\phi: F \rightarrow E$ if $\operatorname{Ext}_{Q_{n}}^{1}(F, E(-1))=0$. This is indeed the case since we proved that $H^{1}\left(Q_{n}, E(t)\right)=0$ for all $t \in \mathbb{Z}$.

Thus we have a morphism $\phi: F \rightarrow E$. The determinant of $\phi$ is a constant, since $c_{1}(F)=c_{1}(E)$, and this constant is non-zero, since the restriction of $\phi$ to $Q_{n-1}$ is an isomorphism. Then $\phi$ is an isomorphism, and the lemma is proved.

The next proposition is the key ingredient of the proof of our main result. Roughly speaking, it uses the lemmas of the previous section to describe the restriction to 7-dimensional quadrics of a complex orthogonal to our collection. We will write $\mathbf{R} \Phi$ for the right-derived functors of a left-exact functor $\Phi$, and similarly for the left-derived $\mathbf{L} \Phi$ of a right-exact functor. Given a subvariety $Z$ of $X$, we will denote by $i_{Z}$ the embedding of $Z$ in $X$.

Proposition 11. Let $E$ be an object of the subcategory ${ }^{\perp} \mathscr{D}$ of $\mathrm{D}^{\mathrm{b}}(X)$. Let $Q \subseteq X$ be a smooth 7-dimensional quadric. Then $\mathbf{L} i_{Q}^{*}(E)$ is a direct sum of shifts of $\mathcal{O}_{Q}(11)$.

Proof. By Lemma 1 there exist $s \in H^{0}(X, \mathcal{S})$ and $\sigma \in H^{0}\left(X, \mathcal{S}_{s}\right)$ such that $Q$ is a component of $\mathscr{Q}=\mathscr{Q}_{\sigma}$, so that $\mathscr{Q}$ is the disjoint union of $Q$ and two additional 7-dimensional quadrics $Q^{\prime}$ and $Q^{\prime \prime}$. Set $j=i_{\mathscr{Q}}$, and let $\iota, \iota^{\prime}, \iota^{\prime \prime}$ be the embedding respectively of $Q, Q^{\prime}$ and $Q^{\prime \prime}$ into $\mathscr{Q}$, so that $i_{Q}=j \circ \iota, i_{Q^{\prime}}=j \circ \iota^{\prime}, i_{Q^{\prime \prime}}=j \circ \iota^{\prime \prime}$. Clearly, since $\mathscr{Q}=Q \sqcup Q^{\prime} \sqcup Q^{\prime \prime}$, for all $t \in \mathbb{Z}$, we have:

$$
\mathcal{O}_{\mathscr{Q}}(t) \simeq \iota_{*}\left(\mathcal{O}_{Q}(t)\right) \oplus \iota_{*}^{\prime}\left(\mathcal{O}_{Q^{\prime}}(t)\right) \oplus \iota_{*}^{\prime \prime}\left(\mathcal{O}_{Q^{\prime \prime}}(t)\right) .
$$

Denote by $\Sigma^{\prime}$ and $\Sigma^{\prime \prime}$ the spinor bundles, respectively on $Q^{\prime}$ and $Q^{\prime \prime}$, and write:

$$
\Sigma_{\mathscr{Q}}=\iota_{*}(\Sigma) \oplus \iota_{*}^{\prime}\left(\Sigma^{\prime}\right) \oplus \iota_{*}^{\prime \prime}\left(\Sigma^{\prime \prime}\right) .
$$

This is a vector bundle of rank 8 on $\mathscr{Q}$ that restricts to the spinor bundle on each of the quadrics $Q, Q^{\prime}$ and $Q^{\prime \prime}$.

Now comes the main goal of this proof, which is to show that:

$$
\begin{array}{ll}
j_{*}\left(\mathcal{O}_{\mathscr{Q}}(t)\right) \in \mathscr{D}, & \text { for } 5 \leq t \leq 10, \\
j_{*}\left(\Sigma_{\mathscr{Q}}(6)\right) & \in \mathscr{D} .
\end{array}
$$


To accomplish this task, we first note that $\left.\mathcal{S}\right|_{\mathscr{Q}} \simeq \mathcal{O}_{\mathscr{Q}} \oplus \mathcal{O}_{\mathscr{Q}}(1) \oplus \Sigma_{\mathscr{Q}}$. Indeed, this is essentially just the statement that the vector representation of $\operatorname{Spin}_{10}$ restricted to $\mathrm{Spin}_{8}$ is the sum of the vector representation and two copies of the trivial representation - there remains to take care of the actions of the center of the Levi subgroups of the parabolics (i.e., to compute $c_{1}$ ) to obtain the precise identity. Then, we consider the Koszul complex of the global section $\sigma$ of $\mathcal{S}_{s}$ :

$$
0 \rightarrow \wedge^{9} \mathcal{S}_{s}^{*} \simeq \mathcal{O}_{X}(-5) \rightarrow \wedge^{8} \mathcal{S}_{s}^{*} \rightarrow \cdots \rightarrow \wedge^{2} \mathcal{S}_{s}^{*} \rightarrow \mathcal{S}_{s}^{*} \rightarrow \mathcal{O}_{X} \rightarrow j_{*}\left(\mathcal{O}_{\mathscr{Q}}\right) \rightarrow 0 .
$$

It is now clear that, to prove (22) and (23) it suffices to prove, respectively, that:

$$
\begin{array}{ll}
\wedge^{p} \mathcal{S}_{s}^{*}(t) \in \mathscr{D} & \text { for } 0 \leq p \leq 9, \\
\wedge^{p} \mathcal{S}_{s}^{*} \otimes \mathcal{S}^{*}(7) \in \mathscr{D}, & \text { for } 0 \leq p \leq 9,
\end{array}
$$

where the degree shift between (23) and (25) is explained by $\mathcal{S}^{*}(7) \simeq \mathcal{S}(6)$.

Let us first focus on (24). Taking exterior powers of (2), and of its dual, we get:

$$
\wedge^{p} \mathcal{S}_{s}^{*} \in\left\langle\wedge^{p} \mathcal{S}^{*}, \wedge^{p-1} \mathcal{S}^{*}, \ldots, \mathcal{O}_{X}\right\rangle .
$$

Note also that $\wedge^{p} \mathcal{S}_{s}^{*} \simeq \wedge^{9-p} \mathcal{S}_{s}(-5)$. We deduce that (24) holds as soon as:

$\wedge^{p} \mathcal{S}^{*}(t) \in \mathscr{D}$, and $\wedge^{p} \mathcal{S}^{*}(p+t-5) \in \mathscr{D}, \quad$ for $0 \leq p \leq 4$ and $5 \leq t \leq 10$.

This last fact is clear for $p=0,1$. Moreover, it follows from Lemma 5 for $p=2$, Lemma 6 for $p=3$ and Lemma 7 for $p=4$.

We now turn to the proof of (25). By the same argument as above, we reduce the statement to:

$$
\wedge^{p} \mathcal{S}^{*}(7) \otimes \mathcal{S}^{*} \in \mathscr{D}, \quad \text { and } \quad \wedge^{p} \mathcal{S}^{*}(p+2) \otimes \mathcal{S}^{*} \in \mathscr{D}, \quad \text { for } 0 \leq p \leq 4 .
$$

This is clear for $p=0$. For $p=1$, it amounts to:

$$
\wedge^{2} \mathcal{S}^{*}(7), \mathcal{S}_{2}^{*}(7), \mathcal{O}_{X}(7), \wedge^{2} \mathcal{S}^{*}(3), \mathcal{S}_{2}^{*}(3), \mathcal{O}_{X}(2) \in \mathscr{D},
$$

which follows from Lemma 5 and Lemma 4 . For $p=2,3,4$, we can apply Corollary 9. and we are done. So, (22) and (23) are proved.

Let us now go back to the proof of the proposition. Consider the following subcategory of $\mathrm{D}^{\mathrm{b}}(Q)$ :

$$
\mathscr{U}=\left\langle\mathcal{O}_{Q}(5), \mathcal{O}_{Q}(6), \Sigma(6), \mathcal{O}_{Q}(7), \mathcal{O}_{Q}(8), \mathcal{O}_{Q}(9), \mathcal{O}_{Q}(10)\right\rangle
$$

What we have to prove is that $\mathbf{L} i_{Q}^{*}(E)$ lies in $\perp \mathscr{U}$. Indeed, by Kap88, we have $\mathrm{D}^{\mathrm{b}}(Q)=\left\langle\mathscr{U}, \mathcal{O}_{Q}(11)\right\rangle$, so that $\perp \mathscr{U}=\left\langle\mathcal{O}_{Q}(11)\right\rangle$. Hence any object of $\perp \mathscr{U}$ is a direct sum of shifts of $\mathcal{O}_{Q}(11)$.

Now note that, by (20), for any $t, q \in \mathbb{Z}$, we have:

$$
\begin{aligned}
\operatorname{Hom}_{\mathrm{D}^{\mathrm{b}}(Q)}\left(\mathbf{L} i_{Q}^{*}(E), \mathcal{O}_{Q}(t)[q]\right) & \simeq \operatorname{Hom}_{\mathrm{D}^{\mathrm{b}}(\mathscr{Q})}\left(\mathbf{L} j^{*}(E), \iota_{*}\left(\mathcal{O}_{Q}(t)\right)[q]\right) \\
& \subseteq \operatorname{Hom}_{\mathrm{D}^{\mathrm{b}}(\mathscr{Q})}\left(\mathbf{L} j^{*}(E), \mathcal{O}_{\mathscr{Q}}(t)[q]\right) \\
& \simeq \operatorname{Hom}_{\mathrm{D}^{\mathrm{b}}(X)}\left(E, j_{*}\left(\mathcal{O}_{\mathscr{Q}}(t)\right)[q]\right),
\end{aligned}
$$

where we have used that $i_{Q}=j \circ \iota$ hence $\mathbf{L} \iota^{*} \circ \mathbf{L} j^{*} \simeq \mathbf{L} i_{Q}^{*}$, and that $\mathbf{L} \iota^{*}$ and $\mathbf{L} j^{*}$ are left adjoint to $\iota_{*}$ and $j_{*}$ respectively. By the same reason, using (21) we get:

$$
\operatorname{Hom}_{\mathrm{D}^{\mathrm{b}}(Q)}\left(\mathbf{L} i_{Q}^{*}(E), \Sigma(t)[q]\right) \subseteq \operatorname{Hom}_{\mathrm{D}^{\mathrm{b}}(X)}\left(E, j_{*}\left(\Sigma_{\mathscr{Q}}(t)\right)[q]\right) .
$$

So, by (22) and (23), the assumption that $E$ lies in ${ }^{\perp} \mathscr{D}$ implies that $\mathbf{L} i_{Q}^{*}(E)$ lies in $\perp \mathscr{U}$. This finishes the proof. 
Next comes the last lemma that we need. Here we give both our original argument that mimics the analogous result for bundle on the projective plane, cf. OSS80, Theorem 3.2.1], and the simpler, more general argument, suggested by the referee.

Lemma 12. Fix a point $x \in X$, and let $E$ be an object of $\mathrm{D}^{\mathrm{b}}(X)$ such that, for any 8-dimensional quadric $Q_{8} \subseteq X$ through $x$, we have:

$$
\mathbf{L} i_{Q_{8}}^{*}(E) \simeq \bigoplus_{k} \mathcal{O}_{Q_{8}}^{r_{k}}[-k],
$$

for some integers $r_{k}$. Then:

$$
E \simeq \bigoplus_{k} \mathcal{O}_{X}^{r_{k}}[-k]
$$

Proof. The argument follows the proof given in OSS80 that any bundle on the projective plane, which is trivial on any line through a given point, must be trivial. This proof applies almost verbatim since the Cayley plane and its dual define a kind of plane projective geometry, and since the argument carries over to derived functors. Let:

$$
\mathcal{B}_{x}=\left\{(y, \ell) \in X \times \mathbb{O P}^{2}, \quad x, y \in Q_{\ell}\right\} .
$$

The second projection $q: \mathcal{B}_{x} \rightarrow \mathbb{O P}^{2}$ maps $\mathcal{B}_{x}$ to $\check{Q}_{x}$, and makes of $\mathcal{B}_{x}$ a locally trivial fibration in smooth 8-dimensional quadrics over $\check{Q}_{x}$. In particular, $\mathcal{B}_{x}$ is smooth of dimension 16. The first projection $p: \mathcal{B}_{x} \rightarrow X$ is birational since a general point of the Cayley plane belongs to a unique $\mathbb{O}$-line $Q_{\ell}$ passing through $x$. Recall also that $q$ has a section $\xi$ defined by $\xi(\ell)=(x, \ell)$ so $p \circ \xi$ is the constant map $x$, while of course $q \circ \xi$ is the identity.

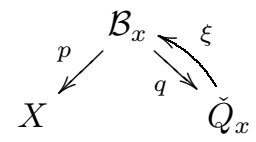

Just as in OSS80, we would like to find a complex $F$ in $\mathrm{D}^{\mathrm{b}}\left(\check{Q}_{x}\right)$ such that:

$$
\mathbf{L} q^{*}(F) \simeq \mathbf{L} p^{*}(E) .
$$

Let us first check that this implies our statement. Note that, by (26), we have:

$$
F \simeq \mathbf{L}(q \circ \xi)^{*}(F) \simeq \mathbf{L} \xi^{*}\left(\mathbf{L} q^{*}(F)\right) \simeq \mathbf{L} \xi^{*}\left(\mathbf{L} p^{*}(E)\right) \simeq \mathbf{L}(p \circ \xi)^{*}(E) \simeq \bigoplus_{k} \mathcal{O}_{\check{Q}_{x}}^{r_{k}}[-k],
$$

where the last isomorphism holds since $p \circ \xi$ has constant value $x$, so $\mathbf{L}(p \circ \xi)^{*}(E)$ is obtained as $(p \circ \xi)^{*}\left(\mathbf{L} i_{x}^{*}(E)\right)$, and clearly $\mathbf{L} i_{x}^{*}(E) \simeq \bigoplus_{k} \mathbb{C}_{x}^{r_{k}}[-k]$, by restriction from $Q_{8}$, since all the $\mathbf{L}^{k} i_{Q_{8}}^{*}(E)$ are free. Hence, we get:

$$
\mathbf{L} p^{*}(E) \simeq \mathbf{L} q^{*}(F) \simeq \bigoplus_{k} \mathcal{O}_{\mathcal{B}_{x}}^{r_{k}}[-k]
$$

and, in turn:

$$
E \simeq \mathbf{R} p_{*}\left(\mathbf{L} p^{*}(E)\right) \simeq \bigoplus_{k} \mathbf{R} p_{*}\left(\mathcal{O}_{\mathcal{B}_{x}}\right)^{r_{k}}[-k] \simeq \bigoplus_{k} \mathcal{O}_{X}^{r_{k}}[-k],
$$

where the last isomorphism is clear, since $p$ satisfies $\mathbf{R} p_{*}\left(\mathcal{O}_{\mathcal{B}_{x}}\right) \simeq \mathcal{O}_{X}$. 
It remains to prove (26). We set:

$$
F=\mathbf{R} q_{*}\left(\mathbf{L} p^{*}(E)\right),
$$

and we have a natural morphism in $\mathrm{D}^{\mathrm{b}}\left(\mathcal{B}_{x}\right)$ :

$$
\varphi: \mathbf{L} q^{*}(F)=\mathbf{L} q^{*}\left(\mathbf{R} q_{*}\left(\mathbf{L} p^{*}(E)\right)\right) \rightarrow \mathbf{L} p^{*}(E) .
$$

We would like to prove that, for any $\ell \in \check{Q}_{x}$, this morphism restricts to an isomorphism in the derived category $\mathrm{D}^{\mathrm{b}}\left(Q_{\ell}\right)$ of the fibre $Q_{\ell}$ of $q$ over $\ell$, and this will finish the proof since these fibres cover $\mathcal{B}_{x}$. Denote by $\alpha$ the embedding of $\{\ell\}$ into $\check{Q}_{x}$, by $\beta$ the restriction of $q$ to $Q_{\ell} \rightarrow\{\ell\}$ and by $\gamma$ the embedding $Q_{\ell} \rightarrow \mathcal{B}_{x}$ so that $p \circ \gamma=i_{Q_{\ell}}$. We have to prove that $\mathbf{L} \gamma^{*}(\varphi)$ is an isomorphism. We write $\mathbf{L} \gamma^{*}(\varphi)$ as:

$$
\mathbf{L} \gamma^{*} \mathbf{L} q^{*} \mathbf{R} q_{*} \mathbf{L} p^{*} E \rightarrow \mathbf{L} \gamma^{*} \mathbf{L} p^{*} E .
$$

On the right-hand side, we have natural isomorphisms:

$$
\mathbf{L} \gamma^{*} \mathbf{L} p^{*} E \simeq \mathbf{L}(p \circ \gamma)^{*} E \simeq \mathbf{L} i_{Q_{\ell}}^{*} E \simeq \bigoplus_{k} \mathcal{O}_{Q_{\ell}}^{r_{k}}[-k]
$$

On the left-hand side of (27), we have natural isomorphisms:

$$
\mathbf{L} \gamma^{*} \mathbf{L} q^{*} \mathbf{R} q_{*} \mathbf{L} p^{*} E \simeq \mathbf{L} \beta^{*} \mathbf{L} \alpha^{*} \mathbf{R} q_{*} \mathbf{L} p^{*} E \simeq \mathbf{L} \beta^{*} \mathbf{R} \beta_{*} \mathbf{L} \gamma^{*} \mathbf{L} p^{*} E \simeq \mathbf{L} \beta^{*} \mathbf{R} \beta_{*} \mathbf{L} i_{Q_{\ell}}^{*} E,
$$

where the middle one is given by smooth base-change. So $\mathbf{L} \gamma^{*}(\varphi)$ is the natural map:

$$
\mathbf{L} \beta^{*} \mathbf{R} \beta_{*} \mathbf{L} i_{Q_{\ell}}^{*} E \rightarrow \mathbf{L} i_{Q_{\ell}}^{*} E,
$$

which is clearly an isomorphism since $\mathbf{L} i_{Q_{\ell}}^{*} E \simeq \bigoplus_{k} \mathcal{O}_{Q_{\ell}}^{r_{k}}[-k]$.

Second proof of Lemma 12, The proof of (26) can be deduced from the following more general fact. Let $Y$ and $S$ be smooth projective varieties, and $q: Y \rightarrow S$ be a flat morphism satisfying $\mathbf{R} q_{*}\left(\mathcal{O}_{Y}\right) \simeq \mathcal{O}_{S}$. For $s \in S$, write $Y_{s}=q^{-1}(\{s\})$ and $i_{s}: Y_{s} \hookrightarrow Y$ the embedding of the fibre $Y_{s}$. Then, an object $F$ of $\mathrm{D}^{\mathrm{b}}(Y)$ such that, for all $s \in S, \mathbf{L} i_{s}^{*}(F)$ lies in $\left\langle\mathcal{O}_{Y_{s}}\right\rangle$ is isomorphic to $\mathbf{L} q^{*}(G)$, for some $G \in \mathrm{D}^{\mathrm{b}}(S)$.

To prove this fact, consider the subcategory $\mathbf{L} q^{*}\left(\mathrm{D}^{\mathrm{b}}(S)\right)^{\perp}$ of $\mathrm{D}^{\mathrm{b}}(Y)$. Since $\mathbf{R} q_{*}$ is right-adjoint to $\mathbf{L} q^{*}$, this category consists of objects $F \in \mathrm{D}^{\mathrm{b}}(Y)$ such that $\mathbf{R} q_{*}(F)=0$, hence we write it as $\operatorname{ker}\left(\mathbf{R} q_{*}\right)$. Thus we have a semiorthogonal decomposition:

$$
\mathrm{D}^{\mathrm{b}}(Y)=\left\langle\operatorname{ker}\left(\mathbf{R} q_{*}\right), \mathbf{L} q^{*}\left(\mathrm{D}^{\mathrm{b}}(S)\right)\right\rangle .
$$

This induces a distinguished triangle:

$$
F^{\prime \prime} \rightarrow F \rightarrow F^{\prime} \rightarrow F^{\prime \prime}[1]
$$

with $\mathbf{R} q_{*}\left(F^{\prime}\right)=0$, and $F^{\prime \prime} \simeq \mathbf{L} q^{*}(G)$ for some $G \in \mathrm{D}^{\mathrm{b}}(S)$. We want to prove that $F^{\prime}=0$ under our assumptions. Assume $F^{\prime} \neq 0$, the object $F^{\prime}$ must restrict non-trivially to some fibre of $q$, say $\mathbf{L} i_{s}^{*}\left(F^{\prime}\right) \neq 0$. Write $q_{s}=\left.q\right|_{Y_{s}}$, set $j_{s}$ for the embedding $\{s\} \hookrightarrow S$, and define $F_{s}=\mathbf{L} i_{s}^{*}\left(F^{\prime}\right)$. By flat base-change:

$$
\mathbf{R} q_{s *}\left(F_{s}^{\prime}\right) \simeq \mathbf{L} j_{s}^{*}\left(\mathbf{R} q_{*}\left(F^{\prime}\right)\right)=0,
$$

so $F_{s}^{\prime}$ sits in $\operatorname{ker}\left(\mathbf{R} q_{s *}\right)$. In other words, $F_{s}^{\prime}$ lies in the subcategory $\left\langle\mathcal{O}_{Y_{s}}\right\rangle^{\perp}$ of $\mathrm{D}^{\mathrm{b}}\left(Y_{s}\right)$, indeed $\mathbf{R} q_{s *}$ is just the derived global section functor $\mathbf{R} \operatorname{Hom}_{Y_{s}}\left(\mathcal{O}_{Y_{s}},-\right)$.

Now, apply $\mathbf{L} i_{s}^{*}$ to (28) to obtain an exact triangle:

$$
\mathbf{L} i_{s}^{*}\left(F^{\prime \prime}\right) \rightarrow \mathbf{L} i_{s}^{*}(F) \rightarrow F_{s}^{\prime} \rightarrow \mathbf{L} i_{s}^{*}\left(F^{\prime \prime}\right)[1] .
$$


In turn, this is the distinguished triangle associated with $F_{s}=\mathbf{L} i_{s}^{*}(F)$ according to the semiorthogonal decomposition:

$$
\mathrm{D}^{\mathrm{b}}\left(Y_{s}\right)=\left\langle\left\langle\mathcal{O}_{Y_{s}}\right\rangle^{\perp}, \mathcal{O}_{Y_{s}}\right\rangle .
$$

So, $F_{s}$ lies in $\left\langle\mathcal{O}_{Y_{s}}\right\rangle$ if and only if $F_{s}^{\prime}=0$, so our assumption contradicts $F_{s}^{\prime} \neq 0$, and the lemma is proved.

\section{Proof of the main Result}

Here we prove that our subcategory $\mathscr{D}$ generates the whole $\mathrm{D}^{\mathrm{b}}(X)$. We have to show that ${ }^{\perp} \mathscr{D}=0$. The idea, inspired by an argument appearing in BO95, is to restrict $E$ to the family of 8 -dimensional quadrics in $X$ through a given point. So let $E$ be a complex of coherent sheaves on $X$, lying in ${ }^{\perp} \mathscr{D}=0$, and let us prove that $E=0$.

Let $x$ be a point of $X$ such that, for all $k, \mathcal{H}^{k}(E)$ is locally free around $x$ (taking $x$ general in $X$ will do). We have:

$$
\left.\mathbf{L} i_{x}^{*}(E) \simeq \bigoplus_{k} \mathbf{L}^{k} i_{x}^{*}(E)[-k] \simeq \bigoplus_{k} \mathcal{H}^{k}(E)\right|_{x}[-k] \simeq \bigoplus_{k} \mathbb{C}_{x}^{r_{k}}[-k]
$$

for some integers $r_{k}$, with $r_{k} \neq 0$ for finitely many $k$ 's.

Let us first use 7-dimensional quadrics. So let $Q_{7}$ be a smooth 7-dimensional quadric contained in $X$ and passing through $x$. In view of Proposition 11, $\mathbf{L} i_{Q_{7}}^{*}(E)$ is the direct sum of shifts of $\mathcal{O}_{Q_{7}}(11)$, so that:

$$
\mathbf{L} i_{Q_{7}}^{*}(E(-11)) \simeq \bigoplus_{k} \mathbf{L}^{k} i_{Q_{7}}^{*}(E(-11))[-k] \simeq \bigoplus_{k} \mathcal{O}_{Q_{7}}^{t_{k}}[-k],
$$

for some (finite) sequence of integers $t_{k}$. All of these cohomology sheaves are free, so restricting from $Q_{7}$ to $x$ each of the $\mathbf{L}^{k} i_{Q_{7}}^{*}(E(-11))$ we must get $\mathbf{L}^{k} i_{x}^{*}(E(-11)) \simeq$ $\mathbb{C}_{x}^{r_{k}}$. Hence $t_{k}=r_{k}$ for each $k$.

Further, note that, given another smooth 7-dimensional quadric $Q_{7}^{\prime} \subseteq X$, meeting $Q_{7}$, we will have:

$$
\mathbf{L} i_{Q_{7}^{\prime}}^{*}(E(-11)) \simeq \bigoplus_{k} \mathbf{L}^{k} i_{Q_{7}^{\prime}}^{*}(E(-11))[-k] \simeq \bigoplus_{k} \mathcal{O}_{Q_{7}^{\prime}}^{r_{k}}[-k],
$$

for the same sequence of integers $r_{k}$. Indeed, the rank of the restricted free sheaves to any point of $Q_{7} \cap Q_{7}^{\prime}$ must agree.

Now let us move to 8-dimensional quadrics. For any smooth such quadric $Q_{8} \subseteq X$ containing $x$, we consider the (finitely many) non-zero sheaves $\mathbf{L}^{k} i_{Q_{8}}^{*}(E)$. We get finitely many proper subschemes of $Q_{8}$ as torsion loci of these sheaves, and we denote by $\tau\left(\mathbf{L}^{k} i_{Q_{8}}^{*}(E)\right)$ the torsion locus of each $\mathbf{L}^{k} i_{Q_{8}}^{*}(E)$. So, we may find a family of smooth hyperplane sections $Q_{7}$ of $Q_{8}$, covering the whole $Q_{8}$, such that, for any given $k, \tau\left(\mathbf{L}^{k} i_{Q_{8}}^{*}(E)\right)$ does not contain $Q_{7}$. Let $f$ be the linear form cutting $Q_{7}$ in $Q_{8}$. We have a long exact sequence:

$$
\begin{aligned}
\cdots & \rightarrow \mathbf{L}^{k-1} i_{Q_{7}}^{*}(E) \rightarrow \mathbf{L}^{k} i_{Q_{8}}^{*}(E(-1)) \stackrel{f}{\rightarrow} \mathbf{L}^{k} i_{Q_{8}}^{*}(E) \\
& \rightarrow \mathbf{L}^{k} i_{Q_{7}}^{*}(E) \rightarrow \mathbf{L}^{k+1} i_{Q_{8}}^{*}(E(-1)) \rightarrow \cdots .
\end{aligned}
$$

Since $\tau\left(\mathbf{L}^{k} i_{Q_{8}}^{*}(E)\right) \not \supset Q_{7}$ for all $k$, all the maps $f=\mathbf{L}^{k} i_{Q_{8}}^{*}(f)$ in the above sequence are injective. So $\left.\mathbf{L}^{k} i_{Q_{7}}^{*}(E) \simeq\left(\mathbf{L}^{k} i_{Q_{8}}^{*}(E)\right)\right|_{Q_{7}}$, and we may split the above long 
sequence into short exact sequences:

$$
0 \rightarrow \mathbf{L}^{k} i_{Q_{8}}^{*}(E(-1)) \stackrel{f}{\rightarrow} \mathbf{L}^{k} i_{Q_{8}}^{*}(E) \rightarrow \mathbf{L}^{k} i_{Q_{7}}^{*}(E) \rightarrow 0 .
$$

Using Proposition 11, this means that, for any $k$, the sheaf $\mathbf{L}^{k} i_{Q_{8}}^{*}(E(-11))$ restricts to $Q_{7}$ as $\mathcal{O}_{Q_{7}}^{r_{k}}$.

We have a similar exact sequence for any other $Q_{7}^{\prime}$ in our covering family of hyperplane sections of $Q_{8}$. Since all these sections $Q_{7}^{\prime}$ obviously meet $Q_{7}$, using again (29) we get that, for any $k$, the sheaf $\mathbf{L}^{k} i_{Q_{8}}^{*}(E(-11))$ restricts to $Q_{7}^{\prime}$ as $\mathcal{O}_{Q_{7}^{\prime}}^{r_{k}}$ (for the same integers $r_{k}$ ). In particular $\mathbf{L}^{k} i_{Q_{8}}^{*}(E(-11))$ is locally free of rank $r_{k}$. Lemma 10 now gives $\mathbf{L}^{k} i_{Q_{8}}^{*}(E(-11)) \simeq \mathcal{O}_{Q_{8}}^{r_{k}}$.

We have thus, for all $k$, an isomorphism $\mathbf{L}^{k^{k}} i_{Q_{8}}(E(-11)) \simeq \mathcal{O}_{Q_{8}}^{r_{k}}$. We would like to conclude that $E(-11)$ is itself isomorphic to $\bigoplus_{k} \mathcal{O}_{X}^{r_{k}}[-k]$. To achieve this, consider any other smooth 8-dimensional quadric $Q_{8}^{\prime}$ contained in $X$. By the same argument as above, we get that, given any $k$, the sheaf $\mathbf{L}^{k} i_{Q_{8}^{\prime}}^{*}(E(-11))$ is free. The rank of this free sheaf must be $r_{k}$, since $Q_{8}$ and $Q_{8}^{\prime}$ meet at $x$, and restricting $\mathbf{L}^{k} i_{Q_{8}^{\prime}}^{*}(E(-11))$ to $x$ must give $\mathbf{L}^{k} i_{x}^{*}(E(-11)) \simeq \mathbb{C}^{r_{k}}$.

We are now in position to apply Lemma 12 to obtain an isomorphism $E(-11) \simeq$ $\bigoplus_{k} \mathcal{O}_{X}^{r_{k}}[-k]$, so that $E \simeq \bigoplus_{k} \mathcal{O}_{X}^{r_{k}}(11)[-k]$. But $E$ belongs to ${ }^{\perp} \mathscr{D}$, in particular $\operatorname{Ext}_{X}^{i}\left(E, \mathcal{O}_{X}(11)\right)=0$ for all $i$. Therefore $r_{k}=0$ for all $k$, so $E=0$. The proof is now complete.

\section{ACKNOWLEDGEMENTS}

The first author would like to thank Institut Fourier at Grenoble for the warm hospitality during his visit at the origin of this work.

Computations were greatly helped by the use of the computer algebra package Lie, see CVL.

The authors wish to thank the referee for several interesting suggestions, and for pointing out an inaccuracy in the first version of this note.

\section{REFERENCES}

[BO95] Alexei I. Bondal and Dmitri O. Orlov, Semiorthogonal decomposition for algebraic varieties, ArXiv e-prints, June 1995.

[CvL] Arjeh M. Cohen and Marc van Leeuwen, Lie, a computer algebra package for lie group computations, Available at http://www-math.univ-poitiers.fr/ maavl/.

[IM05] Atanas Iliev and Laurent Manivel, The Chow ring of the Cayley plane, Compos. Math. 141 (2005), no. 1, 146-160, DOI 10.1112/S0010437X04000788. MR2099773 (2005h:14008)

[IM11] Atanas Iliev and Laurent Manivel, On cubic hypersurfaces of dimension seven and eight, ArXiv e-prints, February 2011.

[Kap88] M. M. Kapranov, On the derived categories of coherent sheaves on some homogeneous spaces, Invent. Math. 92 (1988), no. 3, 479-508, DOI 10.1007/BF01393744. MR939472 (89g:18018)

[Kuz07] Alexander Kuznetsov, Homological projective duality, Publ. Math. Inst. Hautes Études Sci. 105 (2007), 157-220, DOI 10.1007/s10240-007-0006-8. MR2354207 (2008k:14043)

[Kuz08] Alexander Kuznetsov, Lefschetz decompositions and categorical resolutions of singularities, Selecta Math. (N.S.) 13 (2008), no. 4, 661-696, DOI 10.1007/s00029-008-0052-1. MR2403307 (2009h:18022)

[LM03] Joseph M. Landsberg and Laurent Manivel, On the projective geometry of rational homogeneous varieties, Comment. Math. Helv. 78 (2003), no. 1, 65-100. MR.1966752 (2004a:14050) 
[LM04] J. M. Landsberg and L. Manivel, Series of Lie groups, Michigan Math. J. 52 (2004), no. 2, 453-479, DOI 10.1307/mmj/1091112085. MR2069810 (2005f:17009)

[Man11] L. Manivel, On the derived category of the Cayley plane, J. Algebra 330 (2011), 177-187, DOI 10.1016/j.jalgebra.2010.12.011. MR2774623 (2012f:14033)

[OR06] Giorgio Ottaviani and Elena Rubei, Quivers and the cohomology of homogeneous vector bundles, Duke Math. J. 132 (2006), no. 3, 459-508, DOI 10.1215/S0012-7094-06-13233-7. MR2219264(2008b:14075)

[OSS80] Christian Okonek, Michael Schneider, and Heinz Spindler, Vector bundles on complex projective spaces, Progress in Mathematics, vol. 3, Birkhäuser Boston, Mass., 1980. MR.561910 (81b:14001)

[Ott88] Giorgio Ottaviani, Spinor bundles on quadrics, Trans. Amer. Math. Soc. 307 (1988), no. 1, 301-316, DOI 10.2307/2000764. MR.936818 (89h:14012)

[Zak93] F. L. Zak, Tangents and secants of algebraic varieties, Translations of Mathematical Monographs, vol. 127, American Mathematical Society, Providence, RI, 1993. Translated from the Russian manuscript by the author. MR.1234494 (94i:14053)

Université de Pau et des Pays de l'Adour, Avenue de l’Université, BP 576, 64012 PAU Cedex, France

E-mail address: daniele.faenzi@univ-pau.fr

Current address: Université de Bourgogne, Institut de Mathématiques de Bourgogne, CNRS-

UMR 5584, 9 Avenue Alain Savary, BP 47870, 21078 Dijon Cedex, France.

E-mail address: daniele.faenzi@u-bourgogne.fr

$U R L:$ http://univ-pau.fr/ ${ }^{\sim}$ faenzi/

Université de Grenoble, BP 74, 38402 Saint-Martin d'Hères, France

E-mail address: laurent.manivel@ujf-grenoble.fr

Current address: UMI 3457 CNRS/Centre de Recherches Mathémathiques, Université de Montréal, Pavillon André-Aisenstadt, 2920 Chemin de la Tour, Montréal, Québec, H3T 1J4, Canada

E-mail address: laurent.manivel@math.cnrs.fr

$U R L:$ http://www-fourier.ujf-grenoble.fr/ manivel/ 